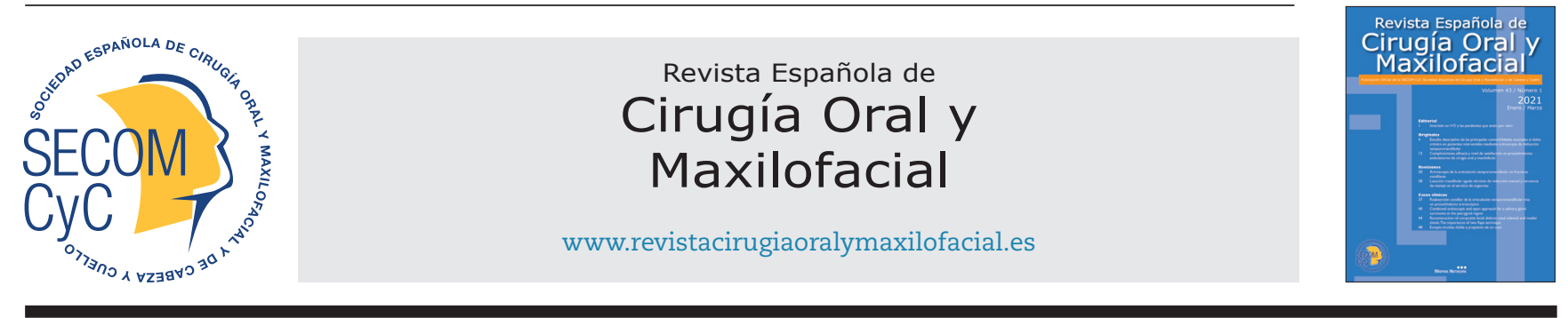

\title{
Revisión
}

\section{Artroscopia de la articulación temporomandibular en fracturas condilares}

\author{
Rafael Martín-Granizo López* \\ Servicio de Cirugía Oral y Maxilofacial, Hospital Clínico San Carlos. Madrid, España
}

INFORMACIÓN DEL ARTÍCULO

Historia del artículo:

Recibido: 25 de marzo de 2021

Aceptado: 29 de marzo de 2021

\section{Palabras clave:}

Articulación temporomandibular, artroscopia, fracturas condilares.

\begin{abstract}
R E S U M E N
Los daños a los tejidos blandos intrarticulares de la articulación temporomandibular (ATM) durante una fractura condilar han sido tradicionalmente relegados y escasamente estudiados. La artroscopia es un procedimiento quirúrgico mínimamente invasivo que puede combinarse con el abordaje abierto de las fracturas condilares con el fin de diagnosticar los daños intrarticulares y tratarlos si se precisa. Tras realizar una revisión bibliográfica, tan solo se han publicado 4 artículos sobre los hallazgos artroscópicos en estos casos y revelan una patología mayor de la esperada, que si no se soluciona puede llevar a secuelas importantes a largo plazo. Se presenta un caso clínico de una fractura subcondilar derecha desplazada en el que se combina una artroscopia de la ATM con un abordaje abierto y reducción de la fractura en el mismo acto. Los hallazgos artroscópicos más comunes son la hemartrosis, los coágulos, el desplazamiento discal, la sinovitis hiperplásica, los daños sobre el fibrocartílago y las perforaciones discales. La presente revisión concluye que es necesario evaluar y diagnosticar los daños intrarticulares en fracturas condilares mandibulares y que la artroscopia es un método adecuado que permite además tratar la patología articular asociada.
\end{abstract}

\section{Arthroscopy of the temporomandibular joint in condylar fractures}

\section{A B S T R A C T}

Damage to the intra-articular soft tissues of the temporomandibular joint (TMJ) during a condylar fracture has traditionally been neglected and poorly studied. Arthroscopy is a minimally invasive surgical procedure that can be combined with the open approach for condylar fractures, in order to diagnose intra-articular damage and treat it if necessary. After conducting a literature review, only 4 articles have been published on arthroscopic findings and they reveal a pathology greater than expected which, if not resolved, can lead to significant long-term sequelae. A clinical case of a displaced right subcondylar fracture is presented in

\footnotetext{
${ }^{*}$ Autor para correspondencia:

Correo electrónico: rmartin.hcsc@salud.madrid.org (Rafael Martín-Granizo López).

DOI: 10.20986/recom.2021.1275/2021
}

1130-0558/C 2021 SECOM. Publicado por Inspira Network. Este es un artículo Open Access bajo la licencia CC BY-NC-ND (http:// creativecommons.org/licenses/by-nc-nd/4.0/). 
which an arthroscopy of the TMJ is combined with an open approach and reduction of the fracture in the same act. The most common arthroscopic findings are hemarthrosis, clots, disc displacement, hyperplastic synovitis, fibrocartilage damage, and disc perforations. The present review concludes that it is necessary to evaluate and diagnose intra-articular damage in mandibular condylar fractures and that arthroscopy is an adequate method that also allows treating associated joint pathology.

\section{INTRODUCCIÓN}

La traumatología de la articulación temporomandibular (ATM) incluye los daños producidos tanto a los tejidos blandos como a las partes óseas, y suelen asociarse. Es cierto que cuando existe tan solo un daño sobre los tejidos blandos, como en el caso del latigazo (hiperextensión-hiperflexión cervical) o whiplash, la tendencia es a minimizarlos en un primer momento, lo que muchas veces conlleva secuelas a largo plazo difíciles de manejar ${ }^{1}$. Por el contrario, cuando existe y se demuestra una fractura ósea asociada, el tratamiento suele instaurarse de manera inmediata, aunque por regla general solamente se centra en la reposición y osteosíntesis de los fragmentos óseos. En estos casos el daño sobre los tejidos no óseos (ligamentos, partes blandas, discomenisco o músculos) suele quedar relegado y puede generar secuelas a largo plazo. Otra causa para un inadecuado diagnóstico de los daños en partes blandas es la dificultad de realizar una RM de urgencias por el tiempo que requiere el estudio o por la escasez de estos aparatos en algunos hospitales ${ }^{2,3}$. Por ello, hoy en día con el avance de los tratamientos quirúrgicos y la posibilidad de terapéuticas multidisciplinares asociadas, se debería de llevar a cabo un manejo integral de estos casos.

En este nuevo contexto puede ser tan importante tratar adecuadamente la fractura ósea como el manejo de los daños a los tejidos blandos. Siempre se debe presuponer que en una situación o un traumatismo que sea capaz de producir una fractura ósea, se va a originar un daño intrarticular en todos los casos. Muchos de ellos podrán solventarse espontáneamente combinando el reposo articular con tratamientos farmacológicos, pero otros necesitarán terapias invasivas sobre la propia articulación.

En casos de sospecha de daño intrarticular, lo primero es diagnosticar el tipo y extensión mediante exploración y pruebas radiológicas, principalmente $\mathrm{RM}$, que generalmente no se solicitan en casos de fracturas condilares ${ }^{2}$. Una vez se obtenga el diagnóstico, el tratamiento incluirá desde técnicas quirúrgicas mínimamente invasivas (infiltraciones intrarticulares de diferentes sustancias, artrocentesis y lavado articular o artroscopia) o técnicas quirúrgicas abiertas sobre la articulación (discopexia), que se pueden combinar con la osteosíntesis de la propia fractura. Lamentablemente, la bibliografía publicada no ofrece ningún protocolo de actuación quirúrgica sobre los tejidos blandos en casos de fracturas condilares, aunque algunos autores sugieren el lavado articular, la movilización precoz y la osteosíntesis de la fractura ${ }^{4}$.

La artroscopia de la ATM es una técnica de cirugía mínimamente invasiva que ofrece buenos resultados (entre un 80 y un $92 \%$ de los casos) con un índice bajo de complicaciones (entre $<1 \%$ y un $5 \%$ de los casos) $)^{5-7}$. Además, es el único procedimiento que permite visualizar en tiempo real la función intrarticular y el aspecto macroscópico de los tejidos. Permite diagnosticar de visu los hallazgos encontrados, biopsiar tejidos y tratar adecuadamente los mismos, dejando la articulación relativamente indemne y funcionante. Además, la infiltración de sustancias beneficiosas, como el ácido hialurónico $(\mathrm{AH})$ o el plasma rico en plaquetas (PRP), ayudará en el proceso reparativo y lubrificante intrarticular. Se debe recordar que siempre que la integridad de una articulación es violada se producen unos mecanismos reparativos que pueden activar fenómenos degenerativos, que serán mayores cuanto mayor sea el daño infringido ${ }^{8}$. Sin embargo, la artroscopia presenta también limitaciones, como son el acceso al espacio articular inferior o el manejo de ciertas patologías degenerativas sobre el fibrocartílago o el disco articular.

El objetivo de la presente revisión es analizar la bibliografía publicada respecto a los daños intrarticulares asociados a las fracturas del cóndilo mandibular y tratar de justificar el empleo de la artroscopia de la ATM como tratamiento complementario de las mismas.

\section{METODOLOGÍA}

Se llevó a cabo una revisión bibliográfica sistemática en las bases de datos PubMed, ScienceDirect y Scopus utilizando las palabras clave arthoscopy, TMJ y condylar fractures. El idioma fue el inglés, incluyendo los años desde 1980 al 2020. Se obtuvieron 11 referencias que fueron filtradas desestimando técnicas quirúrgicas o daños tras whiplash, finalizando con 4 artículos que cumplían los requisitos exigidos de relacionar los hallazgos artroscópicos en fracturas del cóndilo mandibular.

\section{CASO CLÍNICO}

Paciente mujer de 76 años, sin enfermedades ni alergias, que sufrió un accidente doméstico con caída accidental y golpe sin defensa en la zona mentoniana de la mandíbula. La paciente refería dolor en la ATM derecha durante los movimientos mandibulares y maloclusión dentaria. En la OPG de control se evidenció una fractura desplazada y dislocada subcondílea alta derecha. La TC mostró la complejidad de la fractura con un desplazamiento completo, fractura intracapsular con dislocación del fragmento medial fuera de la fosa articular y pérdida de la dimensión vertical de la rama (Figuras 1 y 2). Por ello, se catalogó de una fractura condilar derecha tipo VI-C grado 2 (según la clasificación de Spiessl y Scroll, de 1972, modificada por Loukota y cols. en 2010)9,10, necesitando tratamiento quirúrgico abierto ORIF (Open Reduction and Internal Fixation).

Se planificó realizar previamente a la cirugía abierta y en el mismo acto quirúrgico, una artroscopia de la articulación afecta para valorar los daños intrarticulares y tratarlos adecuadamente. 


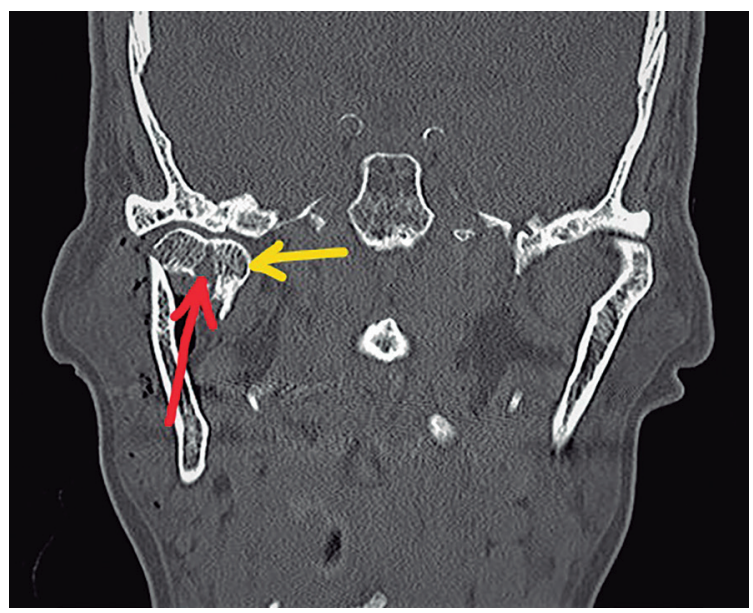

Figura 1. Corte coronal de la TC, donde se aprecia la fractura subcondílea derecha con una fractura intracapsular (flecha roja) con desplazamiento del fragmento medial (flecha amarilla).

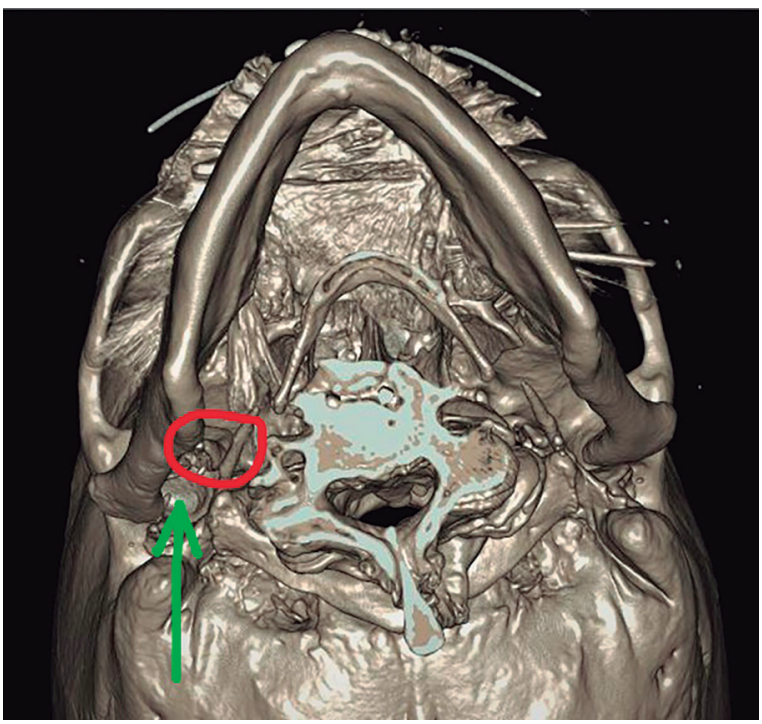

Figura 2. Reconstrucción 3D de la TC con vista caudal, donde se aprecia la dislocación del fragmento medial (círculo rojo) fuera de la fosa articular (flecha verde).

La paciente fue intervenida a los 10 días del traumatismo con intubación nasotraqueal, dibujando las referencias anatómicas, los puntos de entrada de la artroscopia y la incisión para la cirugía abierta (Figura 3). Se comenzó la artroscopia con lisis y lavado de la ATM derecha, realizando un aspirado del espacio articular superior (EAS) que no evidenció hemartrosis, posiblemente por el tiempo transcurrido desde el accidente. A continuación, se infiltró la ATM con anestésico local sin vasoconstrictor para distender el espacio. La entrada al EAS resultó algo más dificultosa al no poder mover el cóndilo y palparse las estructuras óseas, aunque la salida del suero de lavado fue adecuada. Se encontró un importante daño a las estructuras intrarticulares consistente en desgarros del ligamento posterior y fibrosis de la sinovial (Figura 4), posiblemente por un desplazamiento antero-medial completo del disco ar-

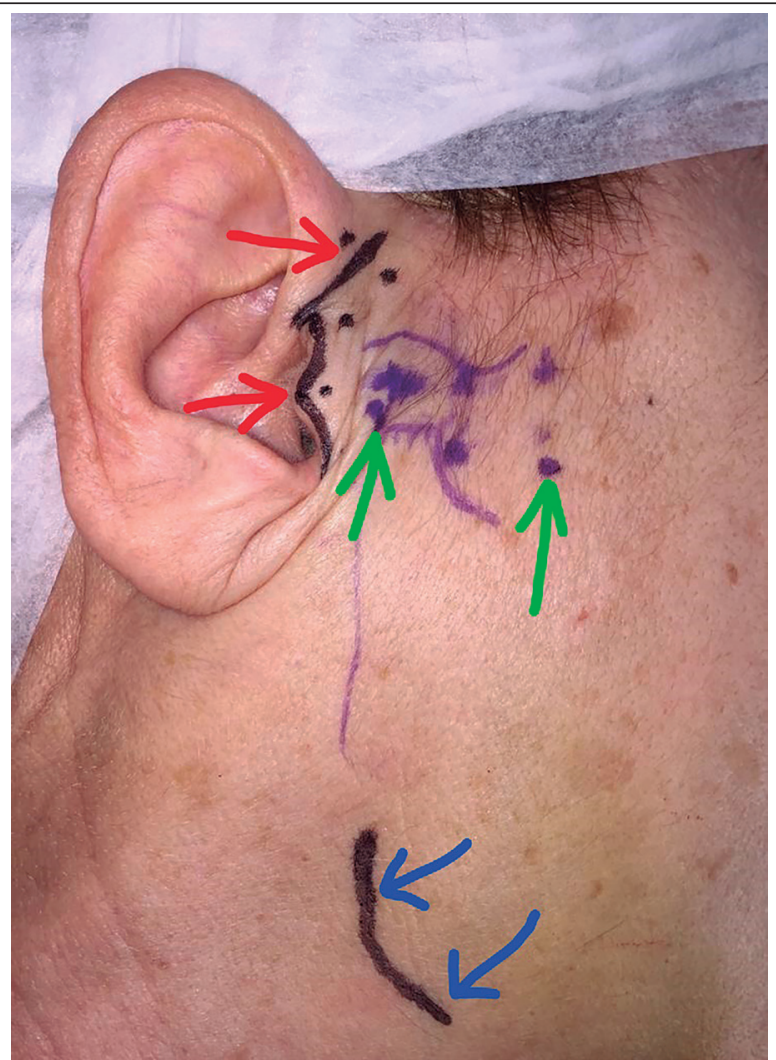

Figura 3. Artroscopia en una fractura subcondilar mandibular izquierda desplazada, previa a su reducción

abierta. Marcado de las estructuras óseas y ángulo mandibular (flechas azules), de los portales de entrada de la artroscopia (flechas verdes) y de la incisión endaural para el abordaje abierto (flechas rojas).

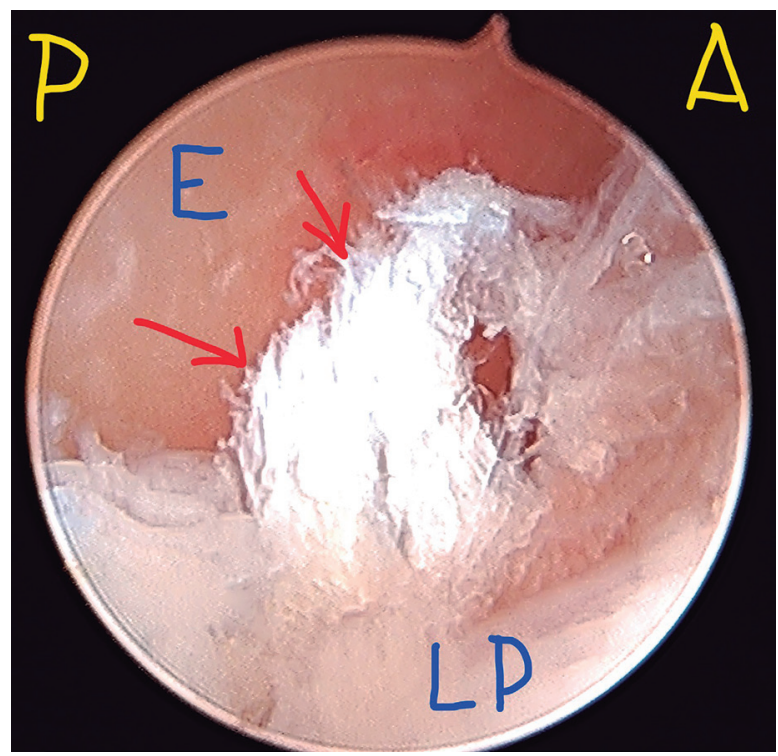

Figura 4. Hallazgos artroscópicos en la fractura condilar. Imagen de un importante daño a las estructuras

intrarticulares con desgarro del ligamento posterior (LP) y fibrosis de la sinovial (flechas rojas) sin evidencia del disco articular, completamente desplazado (A: anterior; P: posterior; E: eminencia articular). 
ticular acompañando al fragmento óseo medial, con un roofing del $0 \%$. También parecía haber un desgarro de la cápsula medial que dejaba al descubierto parte de las fibras musculares del músculo pterigoideo medial (Figura 5). A nivel óseo se observaban áreas de hueso expuesto, tanto en la fosa articular como en la eminencia, por rotura de la integridad del fibrocartílago que lo recubría, junto a zonas con fibrocartílago y áreas de petequias vasculares localizadas posiblemente debido al impacto directo y al traumatismo (Figura 6). También se encontró una perforación discal

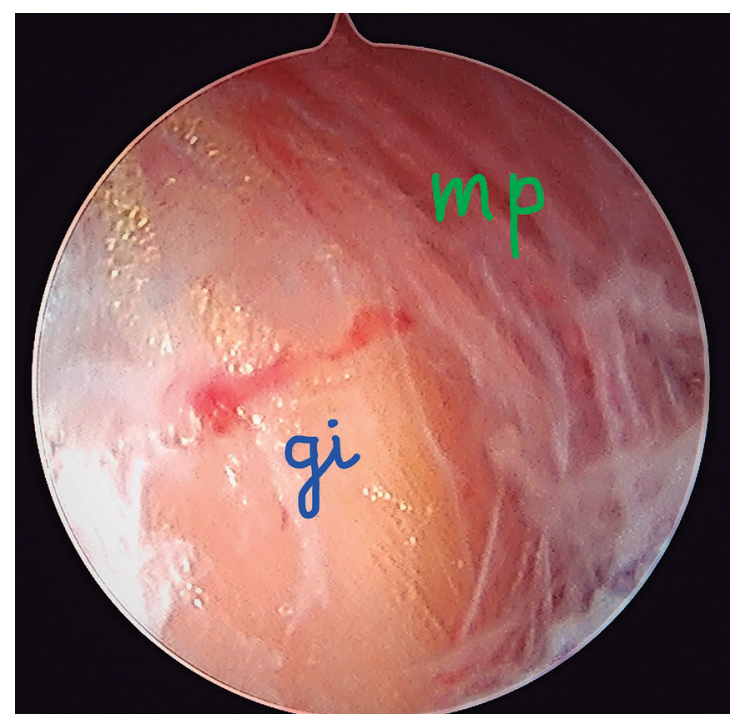

Figura 5. En la exploración de la región medial del EAS parecía existir un desgarro de la cápsula medial que dejaba al descubierto parte de las fibras del músculo pterigoideo (mp) y de la grasa intermuscular (gi).

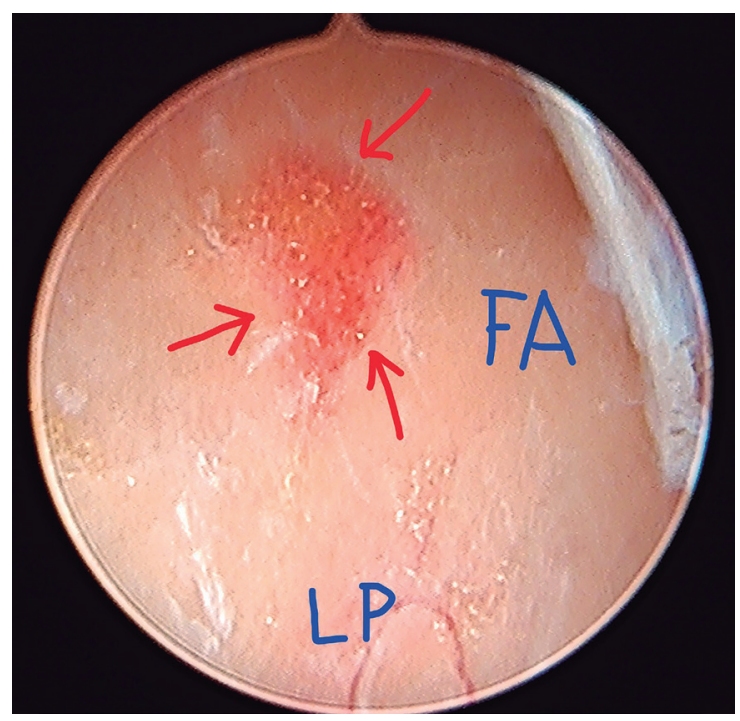

Figura 6. Zonas de hueso expuesto en la fosa articular (FA) por rotura de la integridad del fibrocartílago que lo recubría, junto a un área con fibrocartílago y petequias vasculares (flechas rojas) posiblemente debido al impacto y al traumatismo; se aprecia el comienzo de la inserción del ligamento posterior (LP) en la parte posterior de la fosa articular. aguda en la zona antero-lateral del disco, tal vez provocada por el impacto del cóndilo durante la fractura del mismo (Figura 7). Además, existía un gran colapso de los tejidos con imposibilidad de acceder y explorar el receso anterior del EAS, por lo cual se insertó una segunda aguja de drenaje para mejorar el lavado articular (Figura 8). Finalmente se obtuvo plasma rico en plaquetas (PRP) de la paciente con una fracción rica en plaquetas (PRP-R) de $4 \mathrm{ml}$, de los cuales se infiltraron $2 \mathrm{ml}$ subsinovialmente en el ligamento posterior inflamado mediante la aguja de drenaje (Figura 9) y otros $2 \mathrm{ml}$ intrarticulares libres, una vez finalizado el lavado articular (Figura 10). Estos hallazgos intrarticulares pueden catalogarse como severos y se corresponden con la gravedad encontrada en las peores fracturas en otros estudios ${ }^{11}$.

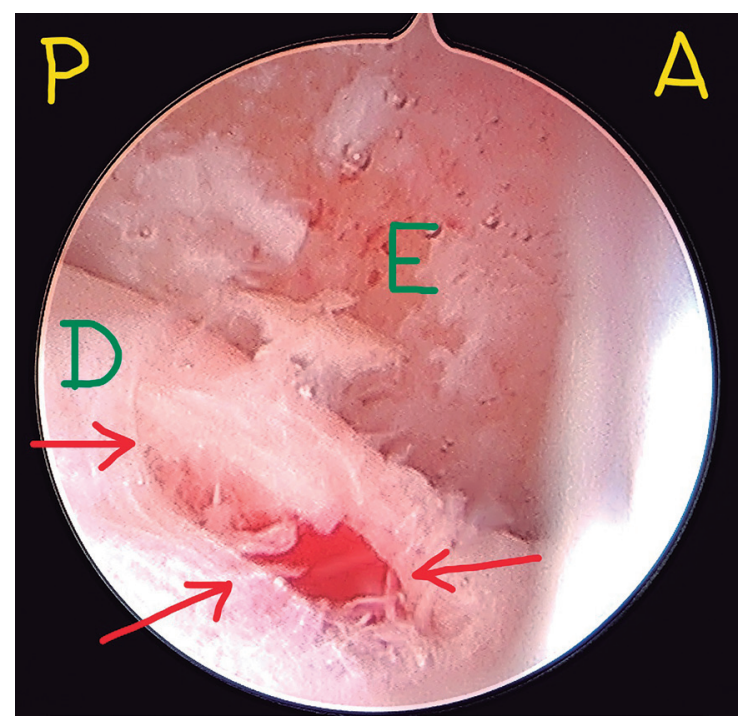

Figura 7. Perforación del disco (D) aguda (flechas rojas) con un hematoma que asoma en el EAI, fibrosis y pérdida del fibrocartílago sobre la eminencia articular (E).

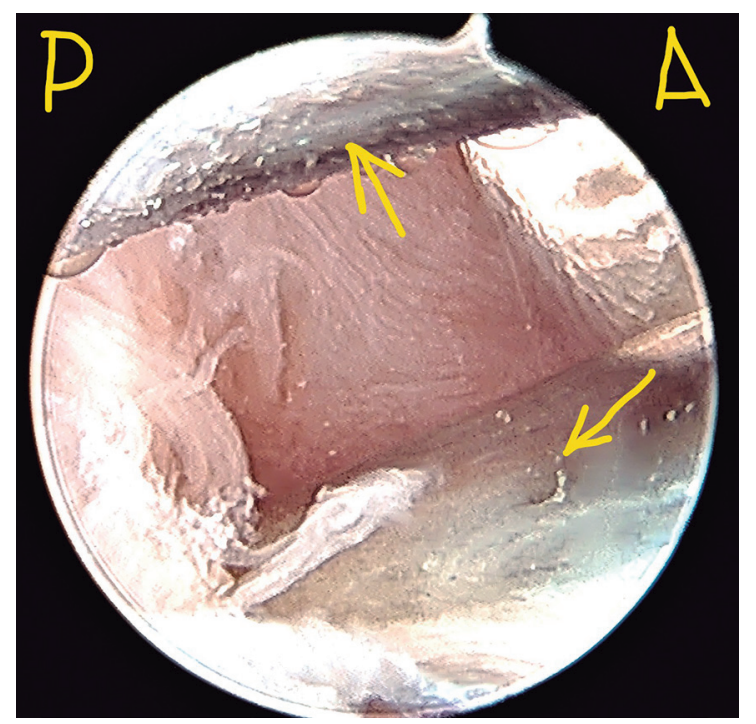

Figura 8. Imagen de la articulación con un gran colapso de los tejidos y las dos agujas de drenaje (flechas amarillas) en el EAS para mejorar el lavado articular. 


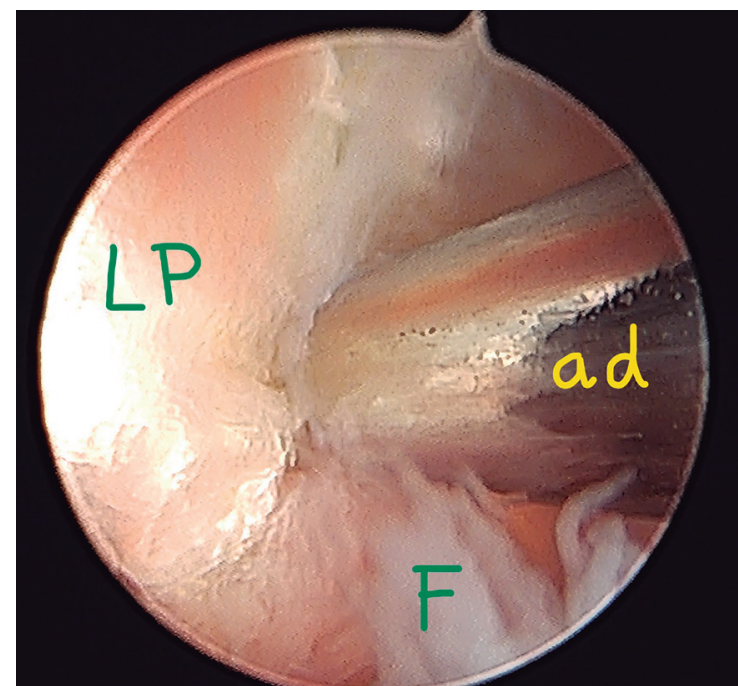

Figura 9. Infiltración de la fracción rica del PRP (PRP-R) subsinovialmente en el ligamento posterior (LP) inflamado mediante la aguja de drenaje (ad) (F: fibrosis del ligamento posterior desgarrado).

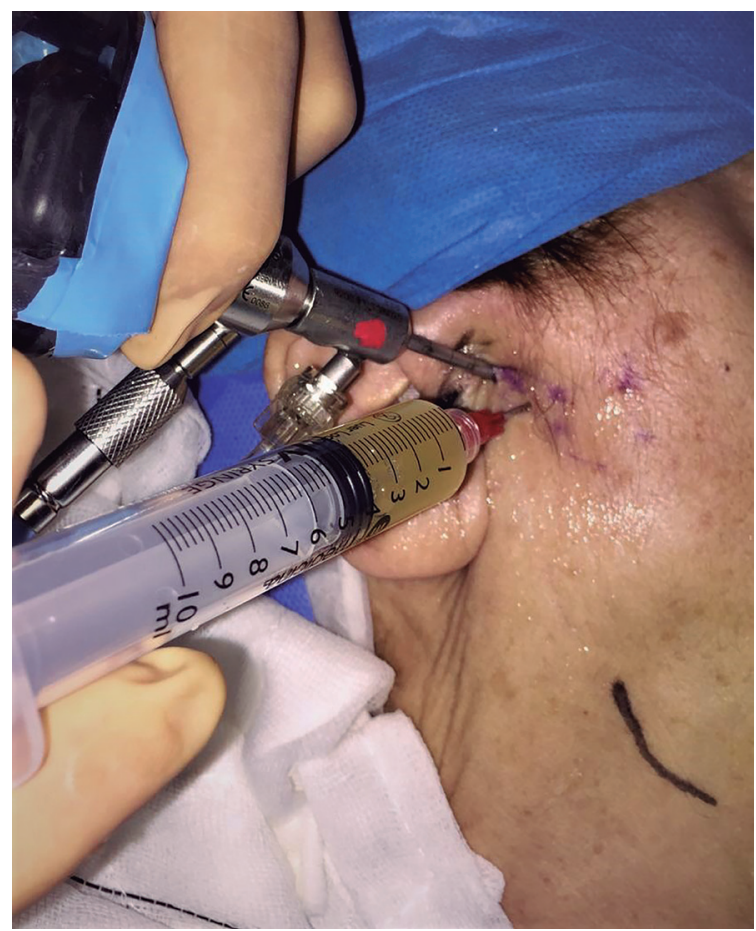

Figura 10. Infiltración de $2 \mathrm{ml}$ de PRP-R en el espacio articular superior a través de la aguja media de drenaje una vez finalizada la artroscopia.

A continuación, se infiltró la incisión abierta con anestésico local y vasoconstrictor y se abordó el espacio articular inferior (EAI) sin abrir el EAS a través de un abordaje endaural y tracción mediante pinza colocada en el ángulo mandibular y máxima relajación muscular. La reducción del fragmento condilar fue muy compleja por la gran dislocación y, tras repo- sicionar el fragmento lateral del cóndilo, se procedió al bloqueo rígido mandibular intraoral mediante 4 tornillos de FIM y se modeló una placa trapezoidal de titanio que se estabilizó con 4 tornillos monocorticales de titanio de 1,5 mm. Finalmente, se infiltraron los tejidos periarticulares con $4 \mathrm{ml}$ del PRP pobre en plaquetas (PRP-P) obtenido previamente y, tras retirar el bloqueo y comprobar la adecuada estabilidad de la fractura, se colocó un drenaje aspirativo y se cerró la herida con sutura monofilamento. La paciente estuvo con dieta líquida 10 días y cobertura antibiótica 7 días, comenzando con ejercicios de movilidad mandibular activa a las 24 horas hasta el límite del dolor. La evolución fue satisfactoria y en la TC de control se objetivó la adecuada reposición del fragmento lateral manteniendo la dimensión vertical (Figura 11). El seguimiento 12 meses después es satisfactorio con una adecuada movilidad mandibular una buena oclusión dentaria y sin dolor asociado de la ATM.

\section{RESULTADOS}

Muy pocos estudios han analizado mediante artroscopia los daños producidos en los tejidos blandos durante una fractura condilar y su importancia en la evolución del caso. Durante una búsqueda de los artículos indexados y publicados sobre el tema, tan solo aparecen 4 referencias, lo cual habla del desconocimiento de esta área.

Un grupo chino en el año 2015 llevó a cabo un estudio sobre 17 articulaciones en 12 pacientes con fracturas condilares intracapsulares en los que se realizó TC y RM, con un seguimiento máximo de 12 meses. Encontraron que la posición discal era muy importante a la hora de favorecer una buena osificación y reparación de los tejidos blandos. Los hallazgos más frecuentemente encontrados eran el desplazamiento

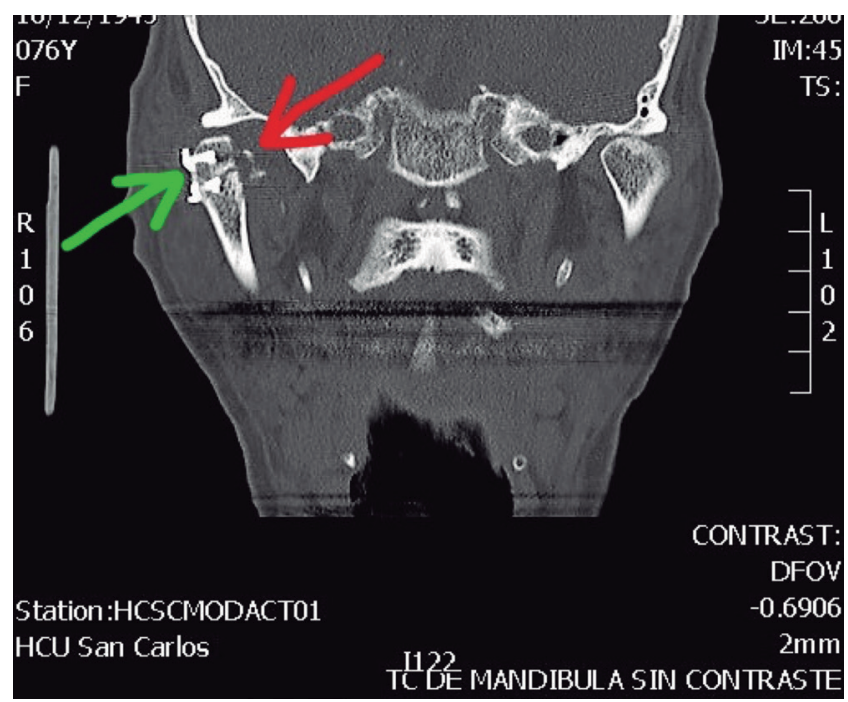

Figura 11. Corte coronal de la TC de control a los 30 días de la cirugía, donde se aprecia la fractura subcondílea bien alineada y fijada (flecha verde) manteniendo la dimensión vertical, con un fragmento desplazado medialmente, aunque con contacto óseo (flecha roja). 
discal acompañando al fragmento óseo desplazado, desgarros en el ligamento posterior y en la cápsula y derrame articular (Tabla I). Estos hallazgos se resolvían espontáneamente en la mayoría de los casos y confirmaban lo que se había sugerido en estudios previos en relación con el papel protector de la integridad discal para evitar anquilosis mandibulares; así, la interposición de tejido discal entre el remanente condilar y la fosa era fundamental para prevenir cambios osteoartríticos y anquilosis. Basándose en estos hallazgos, los autores recomiendan intervenir quirúrgicamente estas articulaciones cuando existan daños en los tejidos blandos, con el fin de reposicionar el disco y los fragmentos óseos y evitar secuelas degenerativas a largo plazo ${ }^{3}$.

El primer artículo publicado fue en el año 1991 por un grupo australiano y ya hablaba de la contraindicación de realizar una artroscopia de la ATM en fracturas condilares, excepto si estas estaban desplazadas, ya que la mayoría evolucionaban sin síntomas articulares ${ }^{12}$. Un estudio previo del mismo grupo realizó una artrografía a estos pacientes y observaron un elevado número de perforaciones discales o discos adheridos, así como adherencias, posiblemente debidas a la fibrosis tras el daño a los tejidos blandos ${ }^{12}$. En otros estudios se ha demostrado que la anquilosis de la ATM es más frecuente cuando existe un daño al disco que en situaciones aparentemente más extremas como en la hemartrosis, pioartrosis, conminución condilar o inmovilización prolongada ${ }^{8}$. Estos autores realizaron una artroscopia con lisis y lavado en 40 articulaciones de 20 pacientes con fracturas condilares que requerían reducción abierta; la edad media era de 25 años con 16:4 a favor de los varones y una media de 5,3 días (entre 2 y 10 días) entre el traumatismo y la cirugía. En 4 casos las fracturas fueron bilaterales, en 11 unilaterales y en 5 solamente del cuerpo mandibular. Evaluaron los cambios encontrados en articulaciones con menos de 5 días desde el traumatismo $(n=22)$ o más de 5 días ( $n=18)$, describiendo los siguientes hallazgos: hemartrosis en el $60 \%$ (82\% del primer grupo y $35 \%$ del segundo), hiperemia en el $12 \%$ (20\% y $6 \%$ ), hemosiderina en el $10 \%$, todas en el grupo de más de 5 días (el $22 \%$ ), no existía malposición discal pero sí daños en forma de desgarros y fibrosis en su superficie (65 \%) y en el fibrocartílago de la fosa (52\%). Las articulaciones más dañadas fueron aquellas con fracturas condilares bilaterales y con dislocación del cóndilo. Curiosamente, en las fracturas unilaterales no dislocadas la articulación no fracturada

Tabla I. Hallazgos inmediatos y en el seguimiento (entre paréntesis) en la RM de la ATM de pacientes con 17 fracturas condilares intracapsulares (Yang y cols., 2015) ${ }^{3}$

Desplazamiento discal

Desgarro del tejido retrodiscal

Ausencia de disco en el resto condilar no fracturado

Desgarro capsular

Derrame articular

Engrosamiento del tejido retrodiscal

Tejido osteoide en el tejido retrodiscal

Neoformación ósea condilar presentaba más alteraciones. Las conclusiones eran que en las articulaciones fracturadas los daños intrarticulares son casi siempre bilaterales, aunque la mayoría tienen una resolución espontánea en unas semanas. La hemartrosis aparentemente se resolvía en un corto espacio de tiempo, aunque dejaba signos residuales de hemosiderina, al contrario que los daños tisulares sobre el disco o la eminencia temporal que permanecían durante más tiempo ${ }^{12}$.

El segundo trabajo preliminar fue realizado simultáneamente, aunque fue publicado un año después en la misma revista. En este estudio se realizó una artroscopia del espacio articular superior en 14 pacientes (15 articulaciones) con sintomatología dolorosa a la palpación e inflamación articular antes del tratamiento cerrado o después de la fijación abierta. Esta diferencia es criticada en la discusión del artículo, ya que la movilización durante la cirugía abierta podría condicionar los posteriores hallazgos en la artroscopia ${ }^{4}$. Encontraron hemartrosis por aspiración en 13 de las 15 ATM y, de ellas, en las realizadas en las primeras 24 horas la sangre era roja y abundante, mientras entre los días 1 y 5 la sangre contenía coágulos, era más oscura y de menor cantidad; esto podría relacionarse con la inflamación articular por el líquido acumulado. En cuanto a los hallazgos artroscópicos, los iniciales incluían hiperemia moderada y proliferación sinovial escasa y tras un día la proliferación aumentaba en forma de vellosidades; además, las equimosis sinoviales eran un hallazgo constante, así como la presencia de grandes coágulos de sangre adheridos a las paredes capsulares. En un caso encontraron un prolapso de la cápsula medial hacia el interior del espacio articular, sin hallar explicación para tal hecho. Los autores se atreven a sugerir que los casos donde el impacto fue en el mentón tenían unas mayores hemartrosis que si eran en el cuerpo mandibular. Ellos apuntan que las articulaciones más inflamadas y dolorosas son las que se asocian a hemartrosis y debería realizarse en ellas un lavado o artroscopia, sobre todo en las que se vayan a inmovilizar. También recomiendan no inmovilizar las articulaciones fracturadas antes de la cirugía, ya que en aquellas que tenían función antes de la artroscopia el índice de hemartrosis era menor ${ }^{4}$.

Hubo que esperar casi tres décadas para ver publicados otros dos estudios. El primero, de un grupo checo y eslovaco, analizó 16 pacientes (19 articulaciones) con fracturas intracapsulares divididas según la clasificación de Neff en tipo A (2), tipo B (16) y tipo C (11 fracturas), realizándoles una artroscopia en el lado afecto previamente al abordaje preauricular abierto. El hallazgo más frecuentemente encontrado (en el $100 \%$ de los casos) fue la hiperemia e hipervascularización del ligamento posterior y de la cápsula medial, seguido de dislocación discal en el $90 \%$ de las articulaciones en todos los casos de fracturas tipo $\mathrm{C}$ con desplazamiento medial del fragmento condilar; en los casos sin desplazamiento del disco la morfología era normal. En cuanto a las consecuencias del impacto directo se encontró en un 72 \% de los casos una erosión del fibrocartílago de la eminencia y un hematoma local con hiperemia reactiva; también se visualizaron pólipos y sinovitis hiperplásica en el 48 \% junto a coágulos en el $35 \%$ y el tamaño de los mismos dependía de la fuerza del impacto más que del tiempo transcurrido. Concluyen que en las fracturas intracapsulares la mayoría de los discos se encuentran desplazados al contrario que en fracturas del cuello o subcondilares, lo cual implicaría 
la necesidad de una reposición discal durante la cirugía abierta y sugieren que previamente se realice una artroscopia con lisis y lavado para diagnosticar los daños intrarticulares y para mejorar la evolución ${ }^{13}$.

El siguiente estudio fue publicado en el 2018 por un autor japonés del grupo del conocido cirujano Kondoh durante su fellowship en Australia en el grupo de Goss, que había publicado el primer trabajo sobre este tema casi 30 años antes. El objetivo era aplicar el concepto de los traumatólogos y ortopedas de realizar una reartroscopia meses después del traumatismo para comprobar los daños, la recuperación de los tejidos y las secuelas. Para ello emplearon un artroscopio ultrafino que permite explorar las articulaciones bajo anestesia local y sedación. Reclutaron 20 pacientes con 27 fracturas condilares intracapsulares con una edad media de 52 años, a los que realizaron la artroscopia en la articulación fracturada durante la primera cirugía y a los 4 meses de evolución, registrando los hallazgos intracapsulares y la evolución clínica. El tiempo entre el traumatismo y la cirugía fue de una media de 12 días $\mathrm{y}$ vieron que los pacientes que eran intervenidos antes tenían mejores resultados clínicos. Según este estudio el grado de daño intrarticular se correlaciona directamente con el grado de desplazamiento vertical del cóndilo fracturado. Los hallazgos incluyeron un $11 \%$ con lesiones sobre el hueso temporal y, además, encontraron perforaciones discales en el 14,8 \% de las articulaciones, y todas ellas ocurrieron en los casos más severos y desplazados de las fracturas intracapsulares (tipo C grado 2). También vieron hiperplasia sinovial y fibrosis en todas las fracturas graves con desplazamiento de fragmentos. Por el contrario, en los casos sin desplazamiento fragmentario los hallazgos fueron hipervascularidad e hiperemia del ligamento posterior discal y en la artroscopia 4 meses después, estos cambios habían desaparecido. Finalmente sugieren reconsiderar en las fracturas graves una reducción quirúrgica abierta y una reposición discal con el fin de evitar fibrosis y adherencias que puedan provocar una anquilosis fibrosa a largo plazo ${ }^{11}$.

\section{DISCUSIÓN}

La muy escasa literatura existente, expuesta en los párrafos precedentes, que aborda el tema de la artroscopia de la ATM en los pacientes con fracturas condilares, hace muy difícil establecer unas recomendaciones firmes con un nivel de evidencia científico alto, por lo que lo expuesto en el presente manuscrito constituye una aproximación preliminar basada en la experiencia personal del autor, así como en lo extraído de las series publicadas con anterioridad, todas ellas de autores con experiencia contrastada en el empleo de los procedimientos artroscópicos de la ATM.

Algunas de las consideraciones técnicas que el autor considera importantes en el uso de la artroscopia de la ATM en el paciente con fractura condílea, son las siguientes:

- En una fractura condilar, la anatomía original de la ATM está modificada o alterada. Dependiendo del grado de desplazamiento óseo, los tejidos blandos (cápsula, ligamentos y disco) se encontrarán en una posición anómala y no esperada.

- La intubación debe ser preferentemente nasotraqueal para poder manipular adecuadamente la mandíbula. Se recomienda colocar los tornillos de bloqueo para la FIM durante la cirugía abierta antes de comenzar la artroscopia, una vez dormido el paciente.

- Es recomendable dibujar las relaciones anatómicas de las estructuras óseas, los puntos de entrada para la infiltración o la artroscopia, así como la incisión para el abordaje abierto si se realiza en el mismo acto (Figura 3).

- La entrada en la articulación debe realizarse inicialmente con una aguja gruesa y una jeringa vacía para aspirar y detectar la presencia o ausencia de hemartrosis.

- La infiltración y expansión articular previa a la artroscopia es, en estos casos, más importante si cabe que en una artroscopia normal, ya que al estar desplazados los tejidos la tendencia será a colapsarse el espacio articular superior y especialmente el receso posterior.

- La maniobra de entrada mediante la cánula con el trócar agudo debe realizarse de la manera habitual, aunque resultará más dificultosa al no poder palpar el movimiento condilar en la fosa, debido a que el cóndilo, por estar fracturado y desplazado, permanecerá inmóvil y no se podrá trasladar anteriormente para aumentar el espacio articular; en un estudio publicado al respecto, sus autores registraron 5 casos en los que no pudieron entrar en la ATM durante la artroscopia (de un total de 40 articulaciones) ${ }^{12}$.

- Una vez dentro, la salida de suero será más complicada por el colapso del espacio y por el daño de los tejidos blandos, pudiendo requerir a veces varias agujas de drenaje (Figura 8).

- El paso del receso posterior al receso anterior es una maniobra técnicamente difícil, al no tener la movilidad del cóndilo y no poder aumentar el espacio en el receso anterior y así trasladar hacia atrás el cóndilo. Por este motivo, la maniobra de triangulación para introducir la cánula de trabajo a veces tendrá que realizarse en el receso posterior en lugar de en el anterior, con el riesgo que esta maniobra conlleva para algunas estructuras, como el oído medio.

- En este tipo de articulaciones siempre se debe prever mayores dificultades técnicas y hallazgos patológicos inesperados y, muchas veces, la artroscopia se convierte en una técnica diagnóstica más que terapéutica. Es fundamental, cuanto menos, realizar un buen lavado articular y planificar la intervención, inicialmente, como una artroscopia lisis y lavado.

- Es recomendable la infiltración intrarticular del espacio superior tras la artroscopia (siempre que no se vaya a abrir posteriormente la articulación durante la cirugía abierta) con alguna sustancia beneficiosa como el ácido hialurónico o el PRP (Figura 10).

- La movilización precoz articular sin carga será fundamental a la hora de minimizar los daños intrarticulares, con el fin de estimular la formación de líquido sinovial y reparar las alteraciones inflamatorias.

En el caso clínico aquí presentado se objetivan unos hallazgos patológicos consistentes con los descritos en la literatura revisada. Así, la artroscopia de la ATM podría ser una técnica adecuada para diagnosticar y tratar los daños intrarticulares asociados, aunque se necesitarían otros estudios para valorar la conveniencia de incluir otros métodos poco invasivos, como las infiltraciones o la artrocentesis. 


\section{CONCLUSIONES}

La artroscopia en casos de fracturas condíleas se puede emplear como método diagnóstico intrarticular eficaz y permite aplicar técnicas terapéuticas sobre los hallazgos patológicos encontrados, pudiendo combinarse con el abordaje abierto de las fracturas condilares, con el fin de diagnosticar los daños intrarticulares y tratarlos si se precisa. Los hallazgos artroscópicos en estos casos revelan una patología mayor de la esperada que, si no se soluciona, puede generar secuelas importantes a largo plazo. Los hallazgos artroscópicos más comunes son la hemartrosis con formación de coágulos, la presencia de desplazamiento discal, sinovitis hiperplásica, daños sobre el fibrocartílago e incluso perforaciones discales.

\section{B I B L I O G R A F Í A}

1. Friedman $\mathrm{MH}$, Weisberg J. The craniocervical connection: A retrospective analysis of 300 whiplash patients with cervical and temporomandibular disorders. Cranio. 2000;18(3):163-7. DOI: 10.1080/08869634.2000.11746128.

2. Pressman BD, Shellock FG, Schames J, Schames M. MR imaging of temporomandibular joint abnormalities associated with cervical hyperextension/hyperflexion (whiplash) injuries. J Magn Reson Imaging. 1992;2(5):569-74. DOI: 10.1002/jmri.1880020515.

3. Yang X, Yao Z, He D, Cai Y, Dong M, Yang C. Does soft tissue injury affect intracapsular condylar fracture healing? J Oral Maxillofac Surg. 2015;73(11):2169-80. DOI: 10.1016/j.joms.2015.05.030.

4. Jones JK, Van Sickels JE. A preliminary report of arthroscopic findings following acute condylar trauma. J Oral Maxillofac Surg. 1991;49(1):55-60. DOI: 10.1016/0278-2391(91)90266-o.
5. González-García R. The current role and the future of minimally invasive temporomandibular joint surgery. Oral Maxillofac Surg Clin North Am. 2015;27(1):69-84. DOI: 10.1016/j. coms.2014.09.006.

6. Tsuyama M, Kondoh T, Seto K, Fukuda J. Complications of temporomandibular joint arthroscopy: A retrospective analysis of 301 lysis and lavage procedures performed using the triangulation technique. J Oral Maxillofac Surg. 2000;58(5):500. DOI: 10.1016/s0278-2391(00)90010-7.

7. González-García R, Rodríguez-Campo FJ, Escorial-Hernández V, Muñoz-Guerra MF, Sastre-Pérez J, Naval-Gías L, et al. Complications of temporomandibular joint arthroscopy: a retrospective analytic study of 670 arthroscopic procedures. J Oral Maxillofac Surg. 2006;64(11):1587-91. DOI: 10.1016/j. joms.2005.12.058.

8. Laskin DM. Role of the meniscus in the etiology of posttraumatic temporomandibular joint ankylosis. Int J Oral Surg. 1978;7(4):340-5. DOI: 10.1016/s0300-9785(78)80106-9.

9. Spiessl B, Schroll K. Spezielle Frakturen- und Luxationslehre. Ein kurzes Handbuch in fünf Bänden. Band I/1 Gesichtsschädel. Leipzig: Georg Thieme Vrelag Eds.; 1972.

10. Loukota RA, Neff A, Rasse M. Nomenclature/classification of fractures of the mandibular condylar head. Br J Oral Maxillofac Surg. 2010;48(6):477-8. DOI: 10.1016/j.bjoms.2009.08.036.

11. Ito K, Yamazaki F, Takahashi K, Nogami S, Kondoh T, Goss A. Relationship between intracapsular fracture patterns and arthroscopic findings. J Oral Maxillofac Surg. 2018;76(7):1510. e1-1510.e12. DOI: 10.1016/j.joms.2018.03.019.

12. Goss AN, Bosanquet AG. The arthroscopic appearance of acute temporomandibular joint trauma. J Oral Maxillofac Surg. 1990;48(8):780-3. DOI: 10.1016/0278-2391(90)90330-5.

13. Hirjak D, Galis B, Beno M, Machon V, Mercuri LG, Neff A. Intraoperative arthroscopy of the TMJ during surgical management of condylar head fractures: A preliminary report. J Craniomaxillofac Surg. 2018;46(12):1989-95. DOI: 10.1016/j. jcms.2017.05.032. 